\title{
Prospective randomized comparison of gastrotomy closure associating tunnel access and over-the-scope clip (OTSC) with two other methods in an experimental ex vivo setting
}

Authors

Institutions
Jean-Michel Gonzalez ${ }^{1}$, Kayoko Saito ${ }^{1}$, Changdon Kang ${ }^{1}$, Mark Gromski ${ }^{1}$, Mandeep Sawhney ${ }^{1}$, Ram Chuttani ${ }^{1}$, Kai Matthes ${ }^{1,2}$

\author{
' Department of Medicine, Division of Gastroenterology, Beth Israel Deaconess Medical Center, Harvard Medical School, \\ Boston, MA, USA \\ 2 Department of Anesthesiology, Boston Children's Hospital, Harvard Medical School, Boston, MA, USA
}

submitted 21. July 2014 accepted after revision 26. August 2014

\section{Bibliography Dol http://dx.doi.org/ 10.1055/s-0034-1390794 Published online: 12.11.2014 Endoscopy International Open 2015; 03: E83-E89 (c) Georg Thieme Verlag KG Stuttgart . New York E-ISSN 2196-9736}

\section{Corresponding author} Jean-Michel Gonzalez, MD Dana 506

Division of Gastroenterology Beth Israel Deaconess Medical Center

Harvard Medical School 330 Brookline Ave. Boston, MA 02215 USA

jmgonza05@yahoo.fr jgonza11@bidmc.harvard.edu
Background: Safe transgastric natural orifice transluminal endoscopic surgery (NOTES) procedures require a reliable closure of the gastrotomy. Recently a novel peritoneal access method via a submucosal tunnel has been described with encouraging preliminary results.

Aim: The aim is to compare a submucosal tunnel access plus over-the-scope clip (OTSC) system for closure with two other closure modalities.

Patients and methods: This is a prospective ex vivo study conducted on 42 porcine stomach models equally randomized into three groups in an academic medical center. The procedures performed in each group included: (1) Tunnel $(6 \mathrm{~cm})$ +endoclips; (2) Knife+balloon dilation access + OTSC; and (3) Tunnel+OTSC. A pressurized airleak test was performed to evaluate the strength of the closure. Stomach volumes, procedure

\section{Introduction}

\section{$\nabla$}

The concept of natural orifice transluminal endoscopic surgery (NOTES) appears to be attractive, but the clinical implementation of this novel approach is not as easily accomplished as expected. Initially, there were an escalating number of publications since the first report in 2004 [1] demonstrating an increasing interest in this field, which straddles the boundaries of interventional endoscopy and surgery. Despite these research efforts and the excitement of investigators, especially during the early phase of NOTES, the clinical application of NOTES has been very hesitant, especially in the United States. One major reason for the declining clinical interest in this "disruptive technology" is the concern about the closure of the access organ to the peritoneum. A simple and safe way for peritoneal access allowing a reliable closure, however, is one of the fundamental preconditions for implementation of NOTES and remains a critical area of research. It is generally agreed that standard endoclips are ineffective in times, number of clips, and incision sizes were also registered.

Results: The mean air-leak pressure was statistically higher in Group 3 than in Groups 1 and 2$95.2 \pm 19.3 \mathrm{mmHg}$ versus $72.5 \pm 35.2$ and $79.0 \pm$ $24.5 \mathrm{mmHg}(P<0.05)$. The gastrotomy creation times for Groups 1, 2, and 3 were $28.0 \pm 10.1,4.3$ \pm 1.4 , and $20.1 \pm 10.6$ minutes, respectively, with significantly lower time in Group $2(P<0.001)$. The closure times were $16.1 \pm 6.1,6.5 \pm 1.2$, and $5.3 \pm 3.0$ minutes, respectively, and significantly longer in the endoclip group $(P<0.001)$. There were no differences in the volumes and the incision sizes among the three groups.

Conclusion: The combination of a submucosal tunnel access and OTSC offers a stronger closure than the other methods studied.

providing a reliable full-thickness closure because of their lack of grip strength and the fact that they only capture mucosa, leading to a weak closure in high pressures. Numerous endoscopic devices for closure of the visceral opening after NOTES procedures have been evaluated [1-13] with various outcomes. To date, many ex vivo or survival animal studies have assessed these different modalities: endoclips [1-4], T-tags [5-7], flexible endostitches [7], flexible endoscopic staplers [7-9], loop anchors $[4,10]$, plicating devices $[11,12]$, and KING closure using endoloop [5,13]. However, they remain mostly technically demanding, depend on availability (prototypes), and still show varying degrees of success. In the indication to close a gastrointestinal wall defect, the overthe-scope clip system (OTSC) (Ovesco Inc., Tubingen, Germany) has clearly demonstrated its efficacy in numerous animal studies, and is increasingly being used in humans to repair digestive perforations of up to $30 \mathrm{~mm}[2,13-21]$.

In the meantime, in order to permit a safer closure in NOTES procedures, a novel approach to 
transgastric peritoneal access has been described. In 2007, Sumiyama et al. [14] created a technique, with a reliable submucosal working space, called submucosal endoscopy with mucosal flap (SEMF) based on the endoscopic submucosal dissection (ESD) technique. This approach was further evaluated by Moyer et al. and Yoshizumi et al. in additional studies $[15,18]$, confirming its ability to obtain safe peritoneal access, ensure secure gastric closure, and achieve acceptable healing of the gastric incision site. The benefit of this procedure is that there is a submucosal "safety zone" that can hamper easy peritoneal leakage post-procedure and only requires closing a mucosal defect, as opposed to a transmural defect. In previous studies, the mucosal incision flap was closed with regular endoclips and, to date, no study has been published comparing the modalities of transgastric closure, which include a submucosal tunnel closed by the OTSC system. At present, two of the techniques compared in our study (except for OTSC closure of a submucosal tunnel) are already used in clinical practice with large human series of OTSC perforation closure $[19,20]$; however, no studies have assessed the qualities of closure with the submucosal tunnel in combination with the OTSC system. Thus, the aim of our study is to compare the effectiveness of this approach with two other different methods that have already demonstrated their reliability: standard access with OTSC closure and ESD tunnel access with standard endoclip closure.

\section{Materials and methods}

$\nabla$

This experimental animal study was conducted in our developmental endoscopy laboratory at Beth Israel Deaconess Medical Center. Ex vivo porcine models numbering 42 and consisting of esophagus, stomach, and duodenum were used. The pig stomachs were washed, prepared, and equally randomized into 3 groups of 14, organized as follows: (1) Submucosal tunnel access with standard endoclip closure; (2) Standard access (knife+balloon dilation) with OTSC closure; and (3) Submucosal tunnel access with OTSC closure. Before starting the procedures, the volume of each stomach was measured by filling it with water.

The primary endpoint was the insufflation pressure required to cause an air leakage at the closure site or a burst of the stomach wall at a location other than the gastrotomy site. The secondary endpoints were the procedure time (gastrotomy, closure, and total time), the number of clips used, and the size of the incisions. The interventions were performed on stomach specimens placed in an EASIE-R simulator (Endosim LLC, Hudson, MA, USA) and using a single-channel gastroscope, Exera II (Olympus America, Center Valley, PA, USA). We used an ERBOTOM ICC 200 (Erbe, Tubingen, Germany) as an electrosurgical generator applying a 120W cut current for the mucosal access and a 60-W cautery current for the dissection. The procedure steps in each group were standardized and conducted as described in the $\bullet$ Fig. 1 diagram.

\section{Standard transgastric access}

The standard access, which is the most commonly described in the literature, was carried out using a hook knife (Olympus America). We first performed a 2-mm, full-thickness incision of the anterior gastric wall, in the horizontal part of the stomach in the preantral region. Then we utilized a CRE dilation balloon, ALLIANCE II (Boston Scientific, Marlborough, MA, USA) mounted on its guidewire, which was introduced from the outside and was inflated up to 3 atmospheres corresponding to an 18-mm diame-

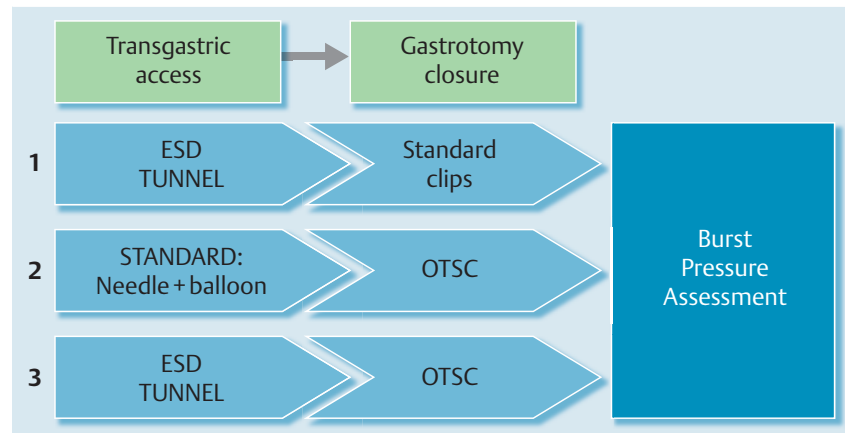

Fig. 1 Diagram showing the three comparison groups.

ter. This inflation was maintained for 1 minute and then the balloon was deflated and the scope was passed through the hole.

\section{Tunnel ESD access}

The submucosal tunnel was created using the hook knife and a plastic cap placed at the tip of the scope. After a submucosal injection of a saline solution mixed with methylene blue in the anterior gastric wall, a first linear mucosal incision was made at one margin of this cushion, situated in all the cases about $2 \mathrm{~cm}$ distal from the gastroesophageal (GE) junction. Then we created a 6-cm tunnel between the submucosa and the muscular layer to reach an exit point at approximately the same anatomical location as the standard access approach (preantral zone). The direction of the dissection was visually controlled from the outside. The length of the tunnel was assessed and confirmed using a plastic injection catheter previously marked with a pen $(7 \mathrm{~cm})$, and introduced through the scope. Once the appropriate length was obtained, a seromuscular, full-thickness incision was performed with the hook knife and the scope was pushed outside the stomach.

\section{Standard clip closure}

The standard clip closure was performed using classical throughthe-scope endoclips, either Olympus Easie-clips 2 (Olympus, Tokyo, Japan), or Boston Resolution clips (Boston Scientific, USA), based upon endoscopist preference. These were placed close and side by side along the incision until achieving a complete and endoscopically satisfying closure and passing an insufflation test.

\section{OTSC clip closure}

We performed the OTSC closure with the assistance of twingrasping forceps (Ovesco). During this step of the procedure, each edge of the incision was grasped with one branch of the for-

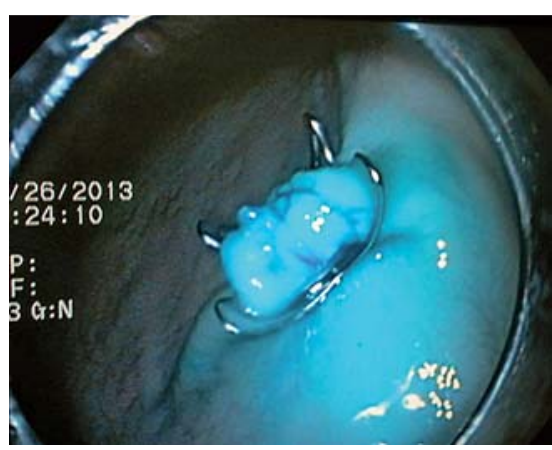

Fig. 2 Closure of the submucosal flap with the over-the-scope clip (OTSC) system (Group 3). 
ceps and then pulled back into the cap in association with suction, and finally the OTSC clip was deployed ( Fig.2). One or more additional regular clips can be placed if the closure is not deemed complete by the endoscopist after a visual and air insufflation inspection.

\section{Outcomes measurements}

\section{Size measurements}

The incision sizes were evaluated at the end of the gastrotomy procedure, just before starting the closure step. The incision size on the serosal side was measured with a ruler, whereas the incision size on the mucosal side was checked by using a specially made, plastic, graduated ruler $(4 \mathrm{~cm})$ introduced into the esophagus, pushed with the scope up to the stomach, and finally grasped with a forceps and placed against the incision to assess its length.

\section{Time measurements}

The time of the gastrotomy was measured from the introduction of the knife into the endoscopic working channel until the endoscope traversed the gastric wall through the access site. The closure time was calculated by starting with the withdrawal of the endoscope from the stomach to load the OTSC (OTSC groups) or with the introduction of the first clip into the working channel (standard clip group), until the closure was achieved and endoscopically satisfying with a good insufflation test.

\section{Air-leak pressure evaluation}

For the evaluation of air-leak pressures, surgical clamps were first applied to the specimen to close the esophagus and the duodenum. Then we placed two needles into the specimen: One was connected to a pump to inflate the stomach with pressurized air, and the other one was plugged into a manometer graduated in millimeters of mercury ( $\mathrm{mmHg}$, used for blood-pressure measurement) to assess the pressure ( $\bullet$ Fig.3). The measurement was carried out by gently inflating the stomach, which was placed into a bowl of water, while checking the pressure on the manometer until we obtained either an air leakage from the incision site (bubbles), or a burst in another part of the stomach (๑ Fig.4).

\section{Sample size and statistical analysis}

The determination of the sample size was based on previously published studies by Von Delius et al. that compared different NOTES gastrotomy closure modalities including tunnel access [21], and Matthes et al., who investigated the OTSC for the closure of standardized perforations using ex vivo models [22]. Those studies included a sample size of 11 and 12 specimens in each group, respectively, to significantly compare closure efficiency of various methods. Based on these results, we estimated a sample size of at least 12 specimens in each group of our study, which had a study design and endpoint similar to the abovementioned studies. To assure sufficient power for statistical comparisons, we decided to include 14 specimens per group.

Data were presented as mean \pm standard deviation and were evaluated for statistical significance by using StatPlus for Macintosh software (AnalystSoft Inc., Alexandria, VA, USA). Statistical comparisons were made among the different groups by using a Student $t$ test and statistical significance was defined as $P \leq 0.05$.

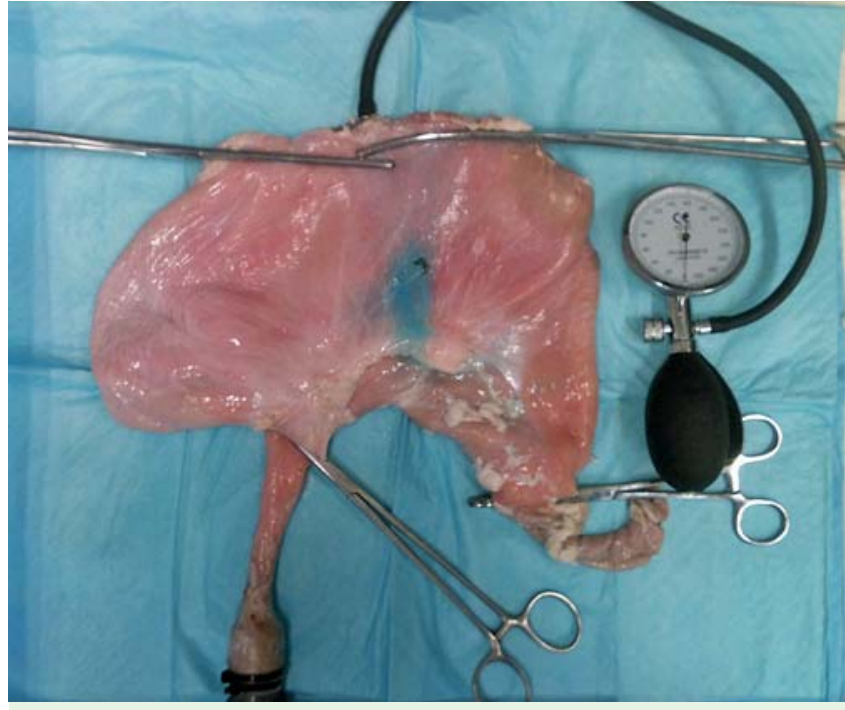

Fig.3 Air-leak pressure evaluation. Surgical clamps close the stomach and the duodenum. Needle connects to the manometer.

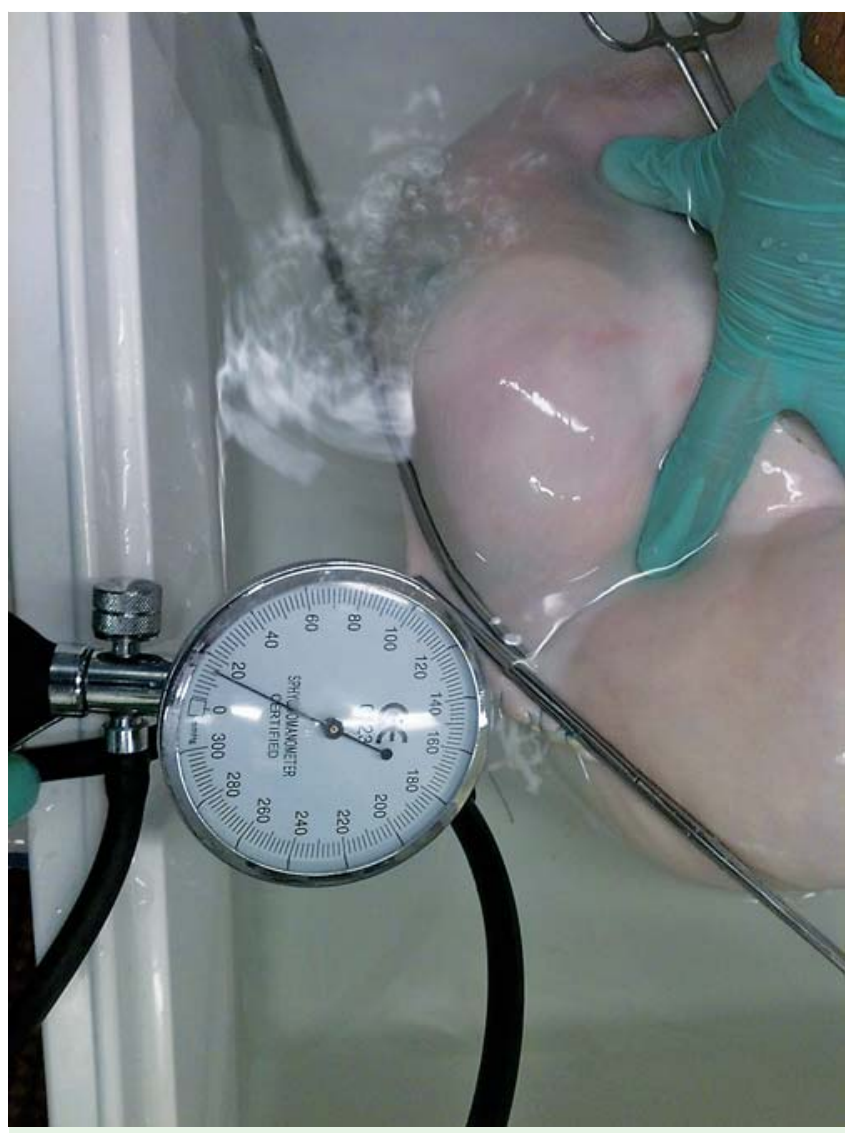

Fig.4 Air-leak measurement procedure with the manometer and the two needles.

\section{Results \\ $\nabla$}

All procedures were successfully completed in each group: Group 1, submucosal tunnel closed by standard endoclips; Group 2, standard transmural gastrotomy closed by the OTSC system; and Group 3, submucosal tunnel closed by the OTSC system. 


\section{Volumes and procedure times}

The mean volumes of the stomachs in each group were $2968 \pm$ $803 \mathrm{~mL}$ (Group 1), $2800 \pm 1124 \mathrm{~mL}$ (Group 2), and $2836 \pm 830 \mathrm{~mL}$ (Group 3), without any significant differences. The procedure times are reported in $\odot$ Table 1, $\odot$ Table 2, and $\odot$ Table 3 . The mean ESD tunnel gastrotomy times were $28 \pm 10$ minutes in Group 1, and $20 \pm 10$ minutes in Group $3(P=0.03)$. The standard transmural gastrotomy access in Group 2, using a hook knife and a dilation balloon, was a faster procedure with a mean duration of $4.25 \pm 1.35$ minutes $(P<0.001)$. Regarding closure, the mean closure times were $16.10 \pm 6.10$ minutes (Group 1), $6.48 \pm 1.24$ minutes (Group 2), and 5.26 \pm 3.03 minutes (Group 3). Closure time in the group with the endoclip closure was significantly longer than in the other two groups that utilized the OTSC closure $(P<0.001)$.

The mean operative total time (gastrotomy + closure) was $44.10 \pm$ 10.10 minutes in Group 1 and was significantly longer $(P<0.001)$ than in Group 3, which was $25.34 \pm 10.28$ minutes. The mean total time in Group 2 was meanwhile significantly shorter $(P<0.001)$ than in the other two groups, with a total time of $11.25 \pm 2.27$ minutes.

\section{Incision sizes-number of clips}

The serosal incision sizes were measured in all the cases, whereas the mucosal incision sizes applied only to the two submucosal tunnel groups (1 and 3 ) because the mucosal and serosal incision sizes are equal in Group 2. The serosal mean incision size was $16.36 \pm 1.34 \mathrm{~mm}, \quad 16.29 \pm 1.27 \mathrm{~mm}$, and $16.33 \pm 2.28 \mathrm{~mm}$ for Groups 1, 2, and 3, respectively, without significant differences among the three groups. Also, there was no statistical difference in the mucosal incision sizes for the groups utilizing the submucosal tunnel technique.

In Group 1 , an average of $7.7 \pm 1.4$ [range $7-12$ clips] clips were required to obtain a satisfactory closure of the defect. In Group 2, 1 single OTSC was required for each closure procedure. In Group 3, an additional standard endoclip was required in one case and two standard endoclips were required in a second case to securely close the defect.

\section{Air-leak pressure}

Group 1-ESD tunnel access + standard clips closure

The mean leakage pressure was $79 \pm 24.5 \mathrm{mmHg}$ [range $40-122$ $\mathrm{mmHg}$. The air leaked through the closure site for 11 cases, and the stomach burst at another location in 3 cases. We also separated the sample into two subgroups according to the mucosal incision size: $\geq 20 \mathrm{~mm}$ and $<20 \mathrm{~mm}$. There were 7 cases in each subgroup and the mean leakage pressure was 75 and $83 \mathrm{mmHg}$, respectively $(P=0.28)$. We encountered two adverse events in this group-small perforations during the tunnel creation. In one case, these didn't affect the 122-mmHg leak pressure, whereas in the other case the $40-\mathrm{mmHg}$ leak pressure did impair performance.

\section{Group 2-needle and dilation access + OTSC closure}

The mean leak pressure for this group was $72.50 \pm 35.18 \mathrm{mmHg}$ [range 20-124 $\mathrm{mmHg}$ ], which was the lowest of the three groups. There was air leakage at the gastrotomy site in 13 cases and a stomach burst in only 1 case. Even in the three procedures

\begin{tabular}{|llll} 
& Group $\mathbf{1}(\mathbf{T}+\mathbf{S t}$ clips) $\mathbf{n = 1 4}$ & Group 2 (St+OTSC) $\mathbf{n = 1 4}$ & P value \\
\hline Volume $(\mathrm{mL})$ & $2968( \pm 803)$ & $2800( \pm 1124)$ & 0.329 \\
\hline Serosal incision size $(\mathrm{mm})$ & $16.36( \pm 1.34)$ & $16.29( \pm 1.27)$ & 0.444 \\
\hline Gastrotomy time $(\mathrm{min})$ & $28.00( \pm 10.10)$ & $4.25( \pm 1.35)$ & $<0.001^{1}$ \\
\hline Closure time $(\mathrm{min})$ & $16.10( \pm 6.16)$ & $6.48( \pm 1.24)$ & $<0.001^{1}$ \\
\hline Total time $(\mathrm{min})$ & $44.10( \pm 10.10)$ & $11.25( \pm 2.27)$ & $<0.001^{1}$ \\
\hline Air-leak pressure $(\mathrm{mmHg})$ & $79( \pm 24.47)$ & $72.50( \pm 35.18)$ & 0.288
\end{tabular}

Table 1 Outcomes comparison between groups 1 and 2 .

Abbreviations: OTSC, over-the-scope clip; St, standard; T, tunnel. ${ }^{1}$ Statistically significant.

\begin{tabular}{|llll|}
\hline & Group $\mathbf{1}(\mathbf{T}+$ St clips) $\mathbf{n = 1 4}$ & Group 3 (T+OTSC) $\mathbf{n = 1 4}$ & P value \\
\hline Volume $(\mathrm{mL})$ & $2968( \pm 803)$ & $2836( \pm 830)$ & 0.338 \\
\hline Mucosal incision size $(\mathrm{mm})$ & $18.07( \pm 2)$ & $16.79( \pm 2.01)$ & 0.056 \\
\hline Serosal incision size $(\mathrm{mm})$ & $16.36( \pm 1.34)$ & $16.33( \pm 2.28)$ & 0.382 \\
\hline Gastrotomy time $(\mathrm{min})$ & $28.00( \pm 10.10)$ & $20.08( \pm 10.57)$ & 0.029 \\
\hline Closure time $(\mathrm{min})$ & $16.10( \pm 6.16)$ & $5.26( \pm 3.03)$ & $<0.001^{1}$ \\
\hline Total time $(\mathrm{min})$ & $44.10( \pm 10.10)$ & $25.34( \pm 10.28)$ & $<0.001^{1}$ \\
\hline Air-leak pressure $(\mathrm{mmHg})$ & $79( \pm 24.47)$ & $95.21( \pm 19.34)$ & $0.031^{1}$ \\
\hline
\end{tabular}

Abbreviations: OTSC, over-the-scope clip; St, standard; T, tunnel.

${ }^{1}$ Statistically significant.

\begin{tabular}{|llll|} 
& Group $\mathbf{2}(\mathbf{S t}+$ OTSC) $\mathbf{n = 1 4}$ & Group $\mathbf{3}(\mathbf{T}+\mathbf{O T S C}) \mathbf{n = 1 4}$ & $\boldsymbol{P}$ value \\
\hline Volume $(\mathrm{mL})$ & $2800( \pm 1124)$ & $2836( \pm 830)$ & 0.462 \\
\hline Serosal incision size $(\mathrm{mm})$ & $16.29( \pm 1.27)$ & $16.33( \pm 2.28)$ & 0.420 \\
\hline Gastrotomy time $(\mathrm{mm})$ & $4.25( \pm 1.35)$ & $20.08( \pm 10.57)$ & $<0.001^{1}$ \\
\hline Closure time $(\mathrm{min})$ & $6.48( \pm 1.24)$ & $5.26( \pm 3.03)$ & 0.069 \\
\hline Total time $(\mathrm{min})$ & $11.25( \pm 2.27)$ & $25.34( \pm 10.28)$ & $0.001^{1}$ \\
\hline Air-leak pressure $(\mathrm{mmHg})$ & $72.50( \pm 35.18)$ & $95.21( \pm 19.34)$ & $0.022^{1}$ \\
\hline
\end{tabular}

Abbreviations: OTSC, over-the-scope clip; St, standard; T, tunnel.

1 Statistically significant.
Table 2 Outcomes comparison between groups 1 and 3 .

Table 3 Outcomes comparison between groups 2 and 3 . 
with the lowest air-leak pressures (20, 24, and $40 \mathrm{mmHg}$ ), endoscopic inspection showed that the clip appeared to be well placed with adequate adaptation to the gastrotomy site. Also, the air insufflation of the stomach via the endoscope did not reveal a visible leak at the closure site. In these cases, the serosal incision sizes where 17,15 , and $18 \mathrm{~mm}$, respectively.

\section{Group 3-ESD tunnel access + OTSC closure}

In this group, the mean air leakage pressure was $95.20 \pm 19.34$ $\mathrm{mmHg}$ [range 50-116 $\mathrm{mmHg}$ ]. There were seven air leakages from the gastrotomy site and seven stomach bursts at locations other than the closure site that were observed at higher insufflation pressures than the other procedures. In our study, the mean insufflation pressure of the subgroup when a burst was observed was $110 \mathrm{mmHg}$ [range $104-116 \mathrm{mmHg}$ ]. This is higher than the subgroup of specimens that leaked at a mean pressure of 80 $\mathrm{mmHg}$ [range 50-104 $\mathrm{mmHg}$ ] before a burst was observed.

We also separated the sample into two subgroups according to the inside incision size: $\geq 17 \mathrm{~mm}(\mathrm{n}=7)$ and $<17 \mathrm{~mm}(\mathrm{n}=7)$. The mean leakage pressure was $90 \pm 22 \mathrm{mmHg}$ and $101 \pm 16 \mathrm{mmHg}$, respectively $(P=0.15)$. In the two cases that needed additional clips, the mucosal incision sizes were 18 and $20 \mathrm{~mm}$. There was only one case of perforation that resulted in an air-leak pressure of $80 \mathrm{mmHg}$, which was slightly lower than the average for this group.

\section{Comparison}

When we compared the three groups in terms of air-leak pressure, there was no statistical difference between Group 1 (tunnel access + standard clips) and Group 2 (standard access + OTSC), 79 $\mathrm{mmHg}$ versus $72.50 \mathrm{mmHg}(P=0.288)$. On the other hand, the leakage pressure in Group 3 (tunnel access+OTSC) was 92.5 $\mathrm{mmHg}$ and significantly higher than both Group $1(P=0.03)$ and Group $2(P=0.02)$. The difference remains significant $(P=0.05)$ if we exclude the failure in Group $1(40 \mathrm{mmHg})$. There were also more stomach bursts in Group 3, which implies that the gastrotomy defects were stronger than other inherent anatomical defects of the specimens.

\section{Discussion \\ $\nabla$}

Gastrotomy closure remains a very challenging aspect of transgastric peritoneal access in NOTES procedures. The consequences of a suboptimal closure are significant, including delayed perforation and peritonitis - all of which could lead to critical illness or death if not detected in a timely manner. Indeed, until endoscopists and minimally invasive surgeons feel confident that their choice of gastrotomy closure is secure and safe, transgastric NOTES procedures will rightfully be relegated to "experimentalonly" cases for its acceptance in human applications.

In our current study, we chose to compare two different methods of closure - a submucosal tunnel access (closed with endoclips and OTSC), and a "standard" access, consisting of knife incision followed by balloon dilation (closed by OTSC). The length of the submucosal tunnel was determined according to previous studies that all demonstrated a reliable closure after SEMF. The mean tunnel lengths were $5 \mathrm{~cm}$ and $8 \mathrm{~cm}$, respectively, so we decided that utilizing a $6-\mathrm{cm}$ tunnel length would be a good compromise between the safety provided by the access, and the difficulty and the time to achieve it. As described for per oral esophageal endoscopic myotomy (POEM) procedures, we employed a submucosal dissection technique for our peritoneal access, despite the higher risk of mucosal injuries. We encountered only a small number of perforation or coagulation injuries. Nevertheless, we believe that the balloon dissection approach, as demonstrated by Sumiyama et al. [5,14], may be safer with a potentially smaller perforation or coagulation risk. Based on our learning experience, we considered this technique for a consecutive in vivo study.

The strength of transluminal closure has been assessed by the endpoint of pressurized leak tests in ex vivo or nonsurvival models. Such tests had already been established as a validated method to assess for closure efficiency in previous studies [7,22,23]. Indeed, even though the entry and exit points of the submucosal tunnel technique are not aligned, it is still a continuous, fullthickness defect traversing all layers of the stomach. We also chose this experimental setup for assessing burst pressures because it provides valuable and objective data about the acute closure strength that is widely used in surgery to evaluate the integrity of digestive anastomosis. We believe this is a valid approach for assessing the closure efficacy of a submucosal tunnel technique. The leak pressure in our standard access group (Group 2) with OTSC closure was consistent with the literature, and particularly with the earlier study of Matthes et al. $(72.5 \pm 35.2 \mathrm{mmHg}$ vs. $74.9 \pm 17.5 \mathrm{mmHg}$ ) [22]. Also in the underlying study, there is a trend toward stronger OTSC closure if the incision size is $<20 \mathrm{~mm}$, but this difference was not statistically significant ( 75 vs. 83 mmHg; $P=0.28$ ). Nevertheless, we agree that there is a probable predictable cut-off issue; however, the use of twin graspers (Ovesco) or tissue anchor (Ovesco) can help to address the size limitation by capturing more tissue. We found that the leak pressure of both groups using the submucosal tunnel technique (Groups 1 and 3) was higher than the standard transgastric access group (although there was no statistical difference between the submucosal tunnel group closed by endoclips and the standard access group). Indeed, the closure in Group 1 (tunnel+endoclips) led to an air-leak pressure of $79 \pm 24 \mathrm{mmHg}$, which is consistent with a study by Von Delius et al. [21] in which they compared the leak pressure of various lengths of submucosal tunnels closed with standard endoclips. In this earlier study, they found an air-leak pressure of $44 \pm 13 \mathrm{mmHg}$ using a 5 -cm tunnel and an air-leak pressure of $87 \pm 19 \mathrm{mmHg}$ using an 8 -cm tunnel. However, the clips were placed manually not endoscopically, and may not represent an accurate clinical scenario. Importantly, we found a statistically higher mean leakage pressure when using the submucosal tunnel technique paired with OTSC closure (95 \pm $19 \mathrm{mmHg}$ ), compared with the other two groups. Moreover, this difference is not affected by the single complication observed (leak pressure $40 \mathrm{mmHg}$ ). In fact, if this case is excluded from the study, the average air-leak pressure increases to $82 \pm 25$ $\mathrm{mmHg}$ in this group; however, the difference between both groups still remains statistically significant $(P<0.001)$. Additionally, in $50 \%$ of the cases, the ending pressure was determined by a burst in the stomach at a site distinct from the closure site. These bursts were observed for higher insufflation pressures that led to a specimen rupture at a location other than the closure site, which indicates a very effective closure-as if there were no defect at all. This phenomenon was not observed in specimens with lower insufflation pressures.

In humans, physiological intragastric pressure is influenced by body mass index, respiration, gender, presence of hiatal hernias, intra-abdominal pressure, and age. Intragastric pressure is usually very low in empty stomachs $(12 \mathrm{mmHg}$ ) [24] but can increase threefold by the presence of liquid or distention, or rise to 90 
$\mathrm{mmHg}$ in the case of coughing or vomiting [25]. Except in one case (leak pressure at $50 \mathrm{mmHg}$ ) the burst pressure of Group 3 $(95 \pm 19 \mathrm{mmHg})$ remained superior to the intragastric pressure whatever the condition (coughing, vomiting), which may give peace of mind to the endoscopist performing the procedure that closure will remain secure despite any potential post-procedural nausea or vomiting.

Comparing the times of the procedures, the standard access gastrotomy was significantly shorter, at 4.3 minutes, compared to the submucosal tunnel times (28.0 and 20.1 minutes). This difference between Groups 1 and 3 for the time of tunnel creation is due to a potential learning curve, since the first six experiments were performed in Group 1.However, at the same time, we did not encounter significantly more mucosal injuries in Group 3 than in Group 1, so we cannot comment on the influence of this difference. Creating a submucosal tunnel requires careful attention to establishing a safe submucosal space; however, even though the tunnel creation may be somewhat more time-consuming in a complicated technique such as NOTES, the trade-off for a stronger gastrotomy closure is likely worth it. Furthermore, it is likely that the indirect submucosal tunnel access (used in Groups 1 and 3) improves healing and reduces the risk of periprocedural complications. It is already used in human applications, such as in POEM procedures. The closure times with the OTSC system (6.5 and 5.3 minutes) were significantly shorter than with the standard clipping technique (16.1 minutes). This was expected because the standard clipping technique requires multiple applications of clips (average of more than seven clips per closure), and in transmural closure it is essential that the clips are fastidiously placed closely adjacent to one another.

There were limitations to this study. First, the two other methods were obviously feasible, strong enough in physiological conditions, and had already been evaluated and demonstrated in patients. However, the aim of our study was to assess if this new approach (tunnel+OTSC) would be more reliable and thereby potentially safer than already-described methods. This was an experimental evaluation that did not take into account secondary fistulas or infection with peritonitis, although we knew that tunnel access is effective in decreasing these risks. One can assume that a stronger closure with higher burst pressures leads to a more reliable closure and thereby potentially decreased risk of closure insufficiency. However, this will have to be verified in survival animal studies and then human studies before drawing definitive conclusions about clinical safety.

Second, this study utilized ex vivo porcine specimens. Thus, we were unable to account for adverse events related to bleeding that can be serious in both the submucosal tunnel technique and also the standard transgastric access technique. Also, we were aware that there are likely more factors than acute closure strength and high burst pressure that contribute to a secure closure of a gastrotomy. Indeed, an adequate tissue perfusion with a minimal wound tension is extremely important, as are surgical closures to avoid tissue ischemia. However, in this current study, we believe that combining submucosal access with closure by OTSC likely mitigates much of those concerns. The OTSC system is applied to the mucosal flap defect only and far from the gastrotomy site, as opposed to the transmural application needed with the standard transgastric approach.

\section{Conclusions}

$\nabla$

In conclusion, the combination of an endoscopic submucosal flap access with an OTSC closure is feasible, quick, and reliable in an ex vivo setting because it has the strongest burst pressure of all modalities studied. This combination provides a stronger gastrotomy sealing than submucosal tunnel with endoclips and standard dilation access with OTSC closure. Additional studies on survival models with pathological assessment should be pursued to further confirm these results prior to applying to human applications.

\section{Competing interests: None}

\section{References}

1 Kalloo AN, Singh VK, Jagannath SB et al. Flexible transgastric peritoneoscopy: a novel approach to diagnostic and therapeutic interventions in the peritoneal cavity. Gastrointest Endosc 2004; 60: 114-117

2 Von Renteln D, Vassiliou MC, Rothstein RI. Randomized controlled trial comparing endoscopic clips and over-the-scope clips for closure of natural orifice transluminal endoscopic surgery gastrotomies. Endoscopy 2009; 41: 1056 - 1061

3 Wagh MS, Merrifield BF, Thompson CC. Survival studies after endoscopic transgastric oophorectomy and tubectomy in a porcine model. Gastrointest Endosc 2006; 63: 473 - 478

4 Desilets DJ, Romanelli JR, Earle DB et al. Loop-anchor purse-string versus endoscopic clips for gastric closure: a natural orifice transluminal endoscopic surgery comparison study using burst pressures. Gastrointest Endosc 2009; 70: 1225 - 1230

5 Sumiyama K, Gostout CJ, Rajan E et al. Endoscopic full-thickness closure of large gastric perforations by use of tissue anchors. Gastrointest Endosc 2007; 65: 134-139

6 Ikeda K, Fritscher-Ravens A, Mosse CA et al. Endoscopic full-thickness resection with sutured closure in a porcine model. Gastrointest Endosc 2005; 62: $122-129$

7 Voermans RP, Worm AM, van Berge Henegouwen MI et al. In vitro comparison and evaluation of seven gastric closure modalities for natural orifice transluminal endoscopic surgery (NOTES). Endoscopy 2008; 40: $595-601$

8 Pham BV, Raju GS, Ahmed I et al. Immediate endoscopic closure of colon perforation by using a prototype endoscopic suturing device: feasibility and outcome in a porcine model (with video). Gastrointest Endosc 2006; 64: 113-119

9 Ryou M, Fong DG, Pai RD et al. Transluminal closure for NOTES : an ex vivo study comparing leak pressures of various gastrotomy and colotomy closure modalities. Endoscopy 2008; 40: 432-436

10 Romanelli JR, Desilets DJ, Chapman CN et al. Loop-anchor purse-string closure of gastrotomy in NOTES(R) procedures: survival studies in a porcine model. Surg Innov 2010; 17: 312-317

11 McGee MF, Marks JM, Onders RP et al. Complete endoscopic closure of gastrotomy after natural orifice translumenal endoscopic surgery using the NDO plicator. Surg Endosc 2008; 22: 214-220

12 McGee MF, Marks JM, Jin J et al. Complete endoscopic closure of gastric defects using a full-thickness tissue plicating device. J Gastrointest Surg 2008; 12: $38-45$

13 Martínek J, Ryska O, Tuckova I et al. Comparing over-the-scope clip versus endoloop and clips (KING closure) for access site closure: a randomized experimental study. Surg Endosc 2013; 27: 1203 -1210

14 Sumiyama K, Gostout CJ, Rajan E et al. Submucosal endoscopy with mucosal flap safety valve. Gastrointest Endosc 2007; 65: 688-694

15 Moyer MT, Pauli EM, Haluck RS et al. A self-approximating transluminal access technique for potential use in NOTES: an ex vivo porcine model (with video). Gastrointest Endosc 2007; 66: 974-978

16 Pauli EM, Moyer MT, Haluck RS et al. Self-approximating transluminal access technique for natural orifice transluminal endoscopic surgery: a porcine survival study (with video). Gastrointest Endosc 2008; 67: $690-697$

17 Mathew A, Tomasko JM, Pauli EM et al. Reliability of gastric access closure with the self-approximating transluminal access technique (STAT) for NOTES. Surg Endosc 2011; 25: 2718-2724

18 Yoshizumi F, Yasuda K, Kawaguchi K et al. Submucosal tunneling using endoscopic submucosal dissection for peritoneal access and closure in 
natural orifice transluminal endoscopic surgery: a porcine survival study. Endoscopy 2009; 41: 707-711

19 Voermans RP, Le Moine O, von Renteln D et al. Efficacy of endoscopic closure of acute perforations of the gastrointestinal tract. Clin Gastroenterol Hepatol 2012; 10: 603-608

20 Kirschniak A, Subotova N, Zieker D et al. The over-the-scope clip (OTSC) for the treatment of gastrointestinal bleeding, perforations, and fistulas. Surg Endosc 2011; 25: 2901-2905

21 Von Delius S, Gillen S, Doundoulakis E et al. Comparison of transgastric access techniques for natural orifice transluminal endoscopic surgery. Gastrointest Endosc 2008; 68: 940 - 947
22 Matthes K, Jung Y, Kato M et al. Efficacy of full-thickness GI perforation closure with a novel over-the-scope clip application device: an animal study. Gastrointest Endosc 2011; 74: 1369-1375

23 Azadani A, Bergström M, Dot $J$ et al. A new in vivo method for testing closures of gastric NOTES incisions using leak of the closure or gastric yield as endpoints. J Laparoendosc Adv Surg Tech Part A 2012; 22: 46 50

$24 \mathrm{El}$-Serag HB, Tran T, Richardson P et al. Anthropometric correlates of intragastric pressure. Scand J Gastroenterol 2006; 41: 887-891

25 Iqbal A, Haider M, Stadlhuber RJ et al. A study of intragastric and intravesicular pressure changes during rest, coughing, weight lifting, retching, and vomiting. Surg Endosc 2008; 22: 2571-2575 